\title{
A singular mutation in the hemagglutinin of the 1918 pandemic virus
}

\author{
Yves-Henri Sanejouand \\ UFIP, CNRS UMR 6286, \\ Faculté des Sciences et des Techniques, \\ Nantes, France.
}

(Dated: October 14, 2016)

PACS numbers: 87.14.E-, $87.19 . x d$

Keywords: influenza - pandemics - hemagglutinin - H1N1

\begin{abstract}
The influenza pandemic of 1918-1919 killed at least 50 million people. The reasons why this pandemic was so deadly remain largely unknown [1]. However, It has been shown that the 1918 viral hemagglutinin allows to reproduce the hallmarks of the illness observed during the original pandemic [2]. Thanks to the wealth of hemagglutinin sequences accumulated over the last decades, amino-acid substitutions that are found in the 1918-1919 sequences but rare otherwise can be identified with high confidence. Such an analysis reveals that Gly188, which is located within a key motif of the receptor binding site, is so rarely found in hemagglutinin sequences that, taken alone, it is likely to be deleterious. Monitoring this singular mutation in viral sequences may help prevent another dramatic pandemic.
\end{abstract}

\section{Introduction}

A dozen years ago, it was shown that introducing the 1918 hemagglutinin (HA) confers enhanced pathogenicity in mice to recent human viruses that are otherwise non-pathogenic in this host. Moreover, like the 1918 one, these recombinant viruses infect the entire lung and induce high levels of macrophage-derived chemokines and cytokines, which results in infiltration of inflammatory cells and severe haemorrhage [2]. In macaques, the whole 1918 virus causes a highly pathogenic respiratory infection that culminates in acute respiratory distress and a fatal outcome [3]. Although the 1918 polymerase genes were found essential for optimal virulence, replacing the 1918 HA by a contemporary human one proved enough for abolishing the lethal outcome of the 1918 virus infection in mices [4], further underlining the key role of the 1918 HA in the deadly process.

The first 1918 HA sequences were obtained in 1999 from formalin-fixed, paraffin-embedded lung tissue samples prepared during the autopsy of victims of the influenza pandemic, as well as from a frozen sample obtained by in situ biopsy of the lung of a victim buried in permafrost since 1918 [5].

Since then, the number of HA sequences determined each year has grown dramatically, jumping from $\approx 100$ in the nineties to $\approx 3,000$ per year nowadays [22]. The goal of the present work is to take advantage of this wealth of data for identifying features that are unique to the 1918 sequence, the underlying hypothesis being that they may prove responsible for the unique behaviour of viruses displaying the 1918 HA.

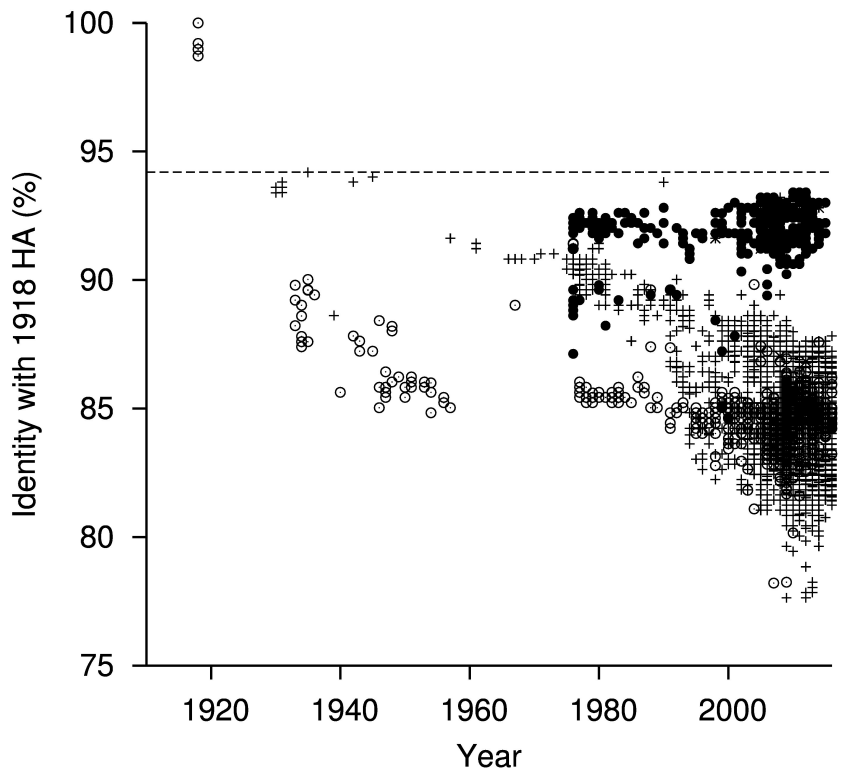

FIG. 1: Percentage of identity of influenza A hemagglutinin sequences with the 1918 sequence, as a function of time. Viruses come from human (open circles), swine (plus symbols), avian (filled circles) or other hosts (stars). The dotted line indicates the highest level of sequence identity observed since 1918 (94.2\%). Results are only shown for the H1 subtype, since other subtypes have sequence identities with the 1918 sequence that are below $75 \%$.

\section{Methods}

52006 HA different protein sequences were retrieved [23] from the NCBI influenza virus resource [6], sequences coming from laboratory viral strains being disregarded. Multiple pairwise sequence alignment was performed using BLAST [7], version 2.2.19, taking as a reference the long $\mathrm{H} 1$ sequence from virus $\mathrm{A} / \mathrm{Thailand} / \mathrm{CU}-\mathrm{MV10} / 2010$. MVIEW [8], version 1.60.1, was used for converting the BLAST output into a standard multiple sequence alignement (MSA). In this MSA, 3365 different amino-acid residues are observed at least 20 times, that is, 6.2 per position along the sequence, on average [24]. Since 1999, a dozen 1918 HA sequences have been determined, at least partially. They differ, at most, by a couple of mutations [5, 9]. One of them was chosen as a representative, namely, the sequence found in PDB structure 4EEF (with $\mathrm{H} 3$ subtype residue numbering) [10]. 


\begin{tabular}{|c|c|c|c|c|c|}
\hline $\begin{array}{l}1918 \mathrm{HA} \\
\text { residue }^{a}\end{array}$ & $\begin{array}{c}\text { Human } \\
\text { H1 }\end{array}$ & $\begin{array}{c}\text { Avian } \\
\text { H1 }\end{array}$ & $\begin{array}{c}\text { Swine } \\
\text { H1 }\end{array}$ & $\begin{array}{l}\text { All } \\
\text { H1 }\end{array}$ & $\begin{array}{l}\text { All } \\
\text { HA }\end{array}$ \\
\hline Gly $188^{b}$ & 0 & 0 & 47 & 47 & 82 \\
\hline $\operatorname{Arg} \mathbf{1 1 6}^{c}$ & 4 & 438 & 162 & 621 & 2071 \\
\hline Ser $159^{b}$ & 17 & 21 & 47 & 85 & 4066 \\
\hline His $285^{b}$ & 17 & 433 & 802 & 1269 & 1297 \\
\hline Ala $219^{b}$ & 22 & 458 & 1642 & 2141 & 5964 \\
\hline Asn $72^{c}$ & 22 & 468 & 289 & 796 & 7489 \\
\hline Lys $\mathbf{1 2 0}^{b}$ & 29 & 446 & 164 & 656 & 10549 \\
\hline $\operatorname{Arg} \mathbf{1 5 3}^{c}$ & 31 & 39 & 418 & 488 & 22855 \\
\hline Ser $264^{b}$ & 45 & 463 & 3562 & 4090 & 9738 \\
\hline Leu $70^{b}$ & 48 & 439 & 2857 & 3367 & 25266 \\
\hline Asp $190^{b}$ & 10055 & 24 & 5340 & 15436 & 28495 \\
\hline Ser $193^{b}$ & 7238 & 312 & 2758 & 10334 & 15936 \\
\hline Lys $\mathbf{2 2 2}^{b}$ & 10871 & 432 & 6101 & 17437 & 23284 \\
\hline Asp $225^{b}$ & 9940 & 12 & 4183 & 14150 & 18093 \\
\hline Total & 11062 & 508 & 6183 & 17794 & 51992 \\
\hline
\end{tabular}

TABLE I: Number of post-1919 hemagglutinin sequences with same residue as the 1918 sequence. Top: residues found in less than 50 human $\mathrm{H} 1$ sequences. Bottom: key residues involved in receptor binding [13]. Bold: residue index of an highly conserved residue, that is, a residue found in more than $95 \%$ of the $\mathrm{H} 1$ sequences. ${ }^{a} \mathrm{H} 3$ subtype residue numbering; ${ }^{b} \mathrm{HA} 1$ subunit; ${ }^{c} \mathrm{HA} 2$ subunit.

\section{Results}

As shown in Figure 1] known HA sequences [25] of post-1919 viruses are all less than $95 \%$ identical to the 1918 one, that is, complete ones differ from the 1918 sequence by at least 25 amino-acid substitutions [26]. Strinkingly, after 1919, HA sequences of human viruses are less than $91.4 \%$ identical to the 1918 HA, a $\approx 90 \%$ level of sequence identity being observed in the thirties as well as more recently, though in a few instances only [27]. On the other hand, HA sequences of avian viruses more than $93 \%$ identical to the 1918 HA have been observed each year since 2005, suggesting that a selection pressure favoring 1918 HA-like sequences is at work in avian species, in line with the hypothesis of an avian origin for the 1918-1919 pandemic [5]. Moreover, among the 41 post-1950 sequences that are more than $93 \%$ identical to the $1918 \mathrm{HA}$, $35(85 \%)$ come from duck species [28], further pinpointing aquatic birds as a possible reservoir [11, 12].

However, the 1918 HA sequence is a singular one. For instance, 17 amino-acid residues in this sequence are found in less than $1 \%$ of other human $\mathrm{H} 1$ sequences. The ten less frequent ones are shown on top of Table I. Most of them are frequent in avian $\mathrm{H} 1$ sequences, or often found [29] in sequences of other HA subtypes (last column).

As a striking exception, after 1919, Gly 188 has not been observed again in human $\mathrm{H} 1$ sequences. It has also not been observed in avian ones. As a matter of fact, it has only been observed in $47 \mathrm{H} 1$ sequences, all of them from swine, a single time in 2003, once each year between 2009 and 2012, and several times each year since then [30]. In human HA of other subtypes, it has been observed 11 times, in sequences from H3N2 or H5N1 viruses. Since 1919, Gly 188 has only been

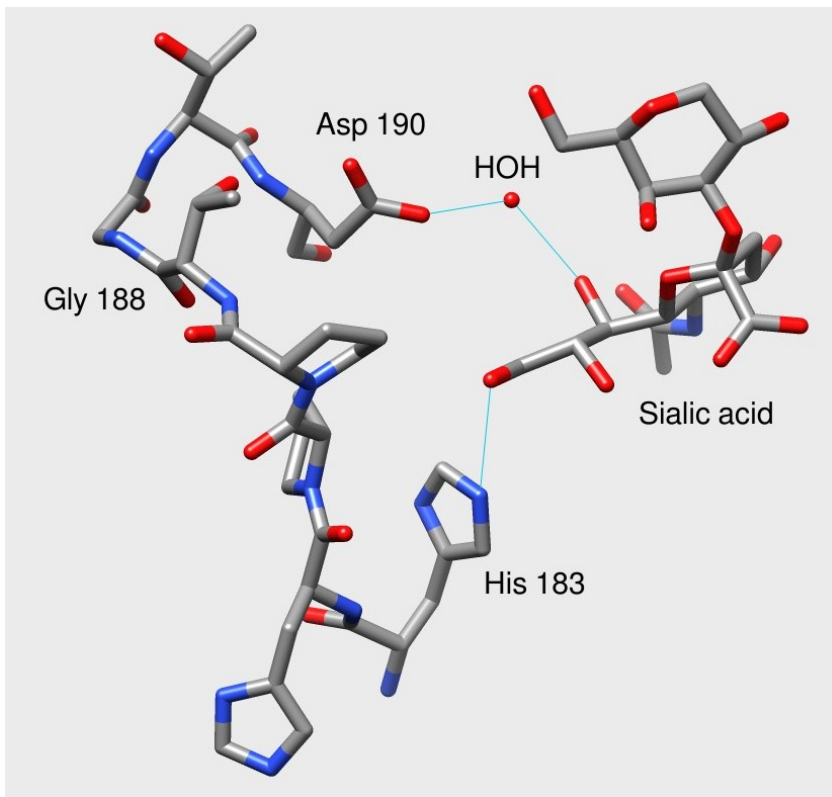

FIG. 2: The 188-hook. Gly 188 is located between two residues interacting either directly (His 183) or through a water molecule (Asp $190)$ with the sialic acid moiety of the hemagglutinin receptor. The conformation shown comes from PDB structure 4JUH [18]. Drawn with UCSF Chimera [19].

observed 82 times, the first time in 2000, in the sequence of an avian H9 HA.

Residue 188 is located at the N-terminus of the "190-helix", which is involved in the HA receptor binding site, but it does not interact directly with the receptor [13, 14]. Three residues are usually observed at this position, namely, serine (41\%), isoleucine $(33 \%)$ and threonine $(21 \%)$. Interestingly, proline which, like glycine, can have a direct impact on the secondary structure [15], the folding [16] or the stability [17] of a protein, is also rarely observed [31]. This suggests that, taken alone, Gly 188 is likely to be deleterious. However, since the 1918 virus proved efficient, one or several compensatory mutations have to be present in its HA sequence.

\section{Discussion}

Why has this mutation been overlooked? After 1919, Gly 188 has not been found again in human $\mathrm{H} 1$ sequences. Overall, it has been found in only $0.2 \%$ of all known HA sequences. The reason why this singular mutation seems to have been overlooked is probably the following one: in the eleven 19181919 HA sequences known so far, Gly 188 has been found ten times. In other words, there is an exception, namely, the HA sequence of virus A/London/1/1918 [9].

How may it be involved in the deadly process ? Gly 188 is located between His 183 and Asp 190, two key residues of the receptor binding site (Figure 2). His 183 is highly conserved, being found in $99.9 \%$ of $\mathrm{H} 1$ sequences. It is thus likely to be involved in the specific recognition of the sialic acid moiety of the HA receptor. On the other hand, Asp 190 is well conserved in H1 HA (88\%) but much less in all HA (56\%), being frequently replaced by Glu $(32 \%)$. This suggests that it is involved in more subtle aspects of the recognition. Indeed, 
a D190E mutation in the 1918 HA results in a preference for the $\alpha 2,3$ sialic acid (avian) receptor [20]. Thus, given the inherent flexibility of Gly residues, introducing Gly at position 188 may allow for alternative orientations of Asp 190 and, as a consequence, for the recognition of other conformations of the sialic acid moiety, or to sialic acid moities linked to disaccharides other than Gal-GlcNac. Though a glycan microarray analysis confirmed a specific recognition of $\alpha 2,6$ sialic acid receptors by 1918 HA, with no binding when GlcNac is absent [21], a change in its repertoire of HA receptors could indeed explain why the 1918 virus proved so deadly.

\section{Conclusion}

Gly 188 has rarely been observed in HA sequences (Table I). Since it is located within a key motif of the HA receptor binding site (Figure 2), this mutation may have an impact on receptor recognition and specificity. This, in turn, could explain why the 1918 HA confers enhanced pathogenicity. It is thus important to check the effects of this mutation on receptor function. Meanwhile, it may prove important to monitor this mutation, noteworthy in swine viruses since it has been observed in swine H1 sequences each year since 2009.
[1] Johnson, NP, Mueller, J (2002) Updating the accounts: global mortality of the 1918-1920 "spanish" influenza pandemic. Bulletin of the History of Medicine 76:105-115.

[2] Kobasa, D et al. (2004) Enhanced virulence of influenza A viruses with the haemagglutinin of the 1918 pandemic virus. Nature 431:703-707.

[3] Kobasa, D et al. (2007) Aberrant innate immune response in lethal infection of macaques with the 1918 influenza virus. $\mathrm{Na}$ ture 445:319-323.

[4] Tumpey, TM et al. (2005) Characterization of the reconstructed 1918 spanish influenza pandemic virus. Science 310:77-80.

[5] Reid, AH, Fanning, TG, Hultin, JV, Taubenberger, JK (1999) Origin and evolution of the 1918 "spanish" influenza virus hemagglutinin gene. Proceedings of the National Academy of Sciences 96:1651-1656.

[6] Bao, Y et al. (2008) The influenza virus resource at the national center for biotechnology information. Journal of virology 82:596-601.

[7] Altschul, SF et al. (1997) Gapped blast and psi-blast: a new generation of protein database search programs. Nucleic acids research 25:3389-3402.

[8] Brown, NP, Leroy, C, Sander, C (1998) Mview: a webcompatible database search or multiple alignment viewer. Bioinformatics 14:380-381.

[9] Reid, AH et al. (2003) 1918 influenza pandemic caused by highly conserved viruses with two receptor-binding variants. Emerging infectious diseases 9:1249-1253.

[10] Whitehead, TA et al. (2012) Optimization of affinity, specificity and function of designed influenza inhibitors using deep sequencing. Nature biotechnology 30:543-548.

[11] Guan, Y et al. (1996) Emergence of avian H1N1 influenza viruses in pigs in china. Journal of virology 70:8041-8046.

[12] Koçer, ZA et al. (2015) Possible basis for the emergence of H1N1 viruses with pandemic potential from avian hosts. Emerging microbes $\mathcal{E}$ infections $4: \mathrm{e} 40$.

[13] Gamblin, S et al. (2004) The structure and receptor binding properties of the 1918 influenza hemagglutinin. Science 303:1838-1842.

[14] Skehel, JJ, Wiley, DC (2000) Receptor binding and membrane fusion in virus entry: the influenza hemagglutinin. Annual review of biochemistry 69:531-569.

[15] Gunasekaran, K, Nagarajaram, H, Ramakrishnan, C, Balaram,
P (1998) Stereochemical punctuation marks in protein structures: glycine and proline containing helix stop signals. Journal of molecular biology 275:917-932.

[16] Krieger, F, Möglich, A, Kiefhaber, T (2005) Effect of proline and glycine residues on dynamics and barriers of loop formation in polypeptide chains. Journal of the American Chemical Society 127:3346-3352.

[17] Nicholson, H, Tronrud, D, Becktel, W, Matthews, B (1992) Analysis of the effectiveness of proline substitutions and glycine replacements in increasing the stability of phage T4 lysozyme. Biopolymers 32:1431-1441.

[18] Zhang, W et al. (2013) Molecular basis of the receptor binding specificity switch of the hemagglutinins from both the 1918 and 2009 pandemic influenza A viruses by a D225G substitution. Journal of virology 87:5949-5958.

[19] Pettersen, EF et al. (2004) UCSF chimera: a visualization system for exploratory research and analysis. Journal of computational chemistry 25:1605-1612.

[20] Glaser, L et al. (2005) A single amino acid substitution in 1918 influenza virus hemagglutinin changes receptor binding specificity. Journal of virology 79:11533-11536.

[21] Stevens, J et al. (2006) Glycan microarray analysis of the hemagglutinins from modern and pandemic influenza viruses reveals different receptor specificities. Journal of molecular biology 355:1143-1155.

[22] According to the NCBI influenza virus resource.

[23] On September $6^{\text {th }}, 2016$.

[24] Positions with a gap in the majority of the sequences were excluded from this statistics.

[25] 37268 sequences were considered for sequence identity comparisons, those with less than 400 residues being disregarded.

[26] The HA sequence in PDB structure 4EEF is 498 residues long.

[27] Four cases in 1976, for instance, the other 13 human virus sequences determined that year being of another subtype (H3).

[28] The six remaining ones come from two other avian species, goose and sparrow, from swine -three cases, the last one being found in the environment.

[29] 1297 cases at least.

[30] 11 times in 2014, 14 times in 2015.

[31] 10 times in all HA sequences. 